\title{
Roles for the Two Cysteine Residues of AhpC in Catalysis of Peroxide Reduction by Alkyl Hydroperoxide Reductase from Salmonella typhimurium ${ }^{\dagger}$
}

\author{
Holly R. Ellis and Leslie B. Poole* \\ Department of Biochemistry, Wake Forest University Medical Center, Winston-Salem, North Carolina 27157 \\ Received June 9, 1997; Revised Manuscript Received August 21, $1997^{\otimes}$
}

\begin{abstract}
The catalytic properties of cysteine residues Cys46 and Cys165, which form intersubunit disulfide bonds in the peroxidatic AhpC protein of the alkyl hydroperoxide reductase (AhpR) system from Salmonella typhimurium, have been investigated. The AhpR system, composed of AhpC and a flavoprotein reductase, $\mathrm{AhpF}$, catalyzes the pyridine nucleotide-dependent reduction of organic hydroperoxides and hydrogen peroxide. Amino acid sequence analysis of the disulfide-containing tryptic peptide demonstrated the presence of two identical disulfide bonds per dimer of oxidized AhpC located between Cys46 on one subunit and Cys165 on the other. Mutant AhpC proteins containing only one (C46S and $\mathrm{C} 165 \mathrm{~S})$ or no $(\mathrm{C} 46,165 \mathrm{~S})$ cysteine residues were purified and shown by circular dichroism studies to exhibit no major disruptions in secondary structure. In NADH-dependent peroxidase assays in the presence of $\mathrm{AhpF}$, the C165S mutant was fully active in comparison with wild-type AhpC, while C46S and C46,165S displayed no peroxidatic activity. In addition, only $\mathrm{C} 165 \mathrm{~S}$ was oxidized by 1 equiv of hydrogen peroxide, giving a species that was stoichiometrically reducible by NADH in the presence of a catalytic amount of $\mathrm{AhpF}$. Oxidized C165S also reacted rapidly with a stoichiometric amount of the thiol-containing reagent 2-nitro-5-thiobenzoic acid to generate a mixed disulfide, and was susceptible to inactivation by hydrogen peroxide, strongly supporting its identification as a cysteine sulfenic acid (Cys46-SOH). The lack of reactivity of the $\mathrm{C} 46 \mathrm{~S}$ mutant toward peroxides was not a result of inaccessibility of the remaining thiol as demonstrated by its modification with 5,5'-dithiobis(2-nitrobenzoic acid), but could be due to the lack of a proximal active-site base which would support catalysis through proton donation to the poor $\mathrm{RO}^{-}$ leaving group. Our results clearly identify Cys46 as the peroxidatic center of AhpC and Cys165 as an important residue for preserving the activity of wild-type AhpC by reacting with the nascent sulfenic acid of the oxidized protein (Cys46-SOH) to generate a stable disulfide bond, thus preventing further oxidation of Cys46-SOH by substrate.
\end{abstract}

The alkyl hydroperoxide reductase system from Salmonella typhimurium protects the cell from the toxic effects of reactive oxygen species generated during normal aerobic metabolism and during times of oxidative stress $(1-3)$. The system consists of two proteins, $\mathrm{AhpF}$ and $\mathrm{AhpC}$, that together catalyze pyridine nucleotide-dependent reduction of organic hydroperoxides and $\mathrm{H}_{2} \mathrm{O}_{2}(2,4-6)$. The genes encoding these two proteins are located on the same operon and are under the transcriptional control of the global stress regulator OxyR (3, 7). Our previous studies have demonstrated that the $57 \mathrm{kDa} \mathrm{AhpF}$ is a thioredoxin reductase-like pyridine nucleotide-dependent protein that contains two redox-active disulfide centers and one FAD per subunit (46). The $21 \mathrm{kDa} A h p C$ protein contains two redox-active cystine disulfide bonds per dimer that have been implicated in the reduction of organic hydroperoxides $(4-6)$.

On the basis of static reductive titrations, AhpF- and AhpC-dependent catalytic assays, and the observed sensitivity of each of the reduced proteins to inactivation by thiol

$\dagger$ This research was supported by NIH Grant GM-50389 and Council for Tobacco Research Awards SA006 and 4501 to L.B.P.

* Author to whom correspondence should be addressed at the Department of Biochemistry, Wake Forest University Medical Center, Medical Center Blvd., Winston-Salem, NC 27157. Telephone: 910716-6711. FAX: 910-716-7671. E-mail: lbpoole@bgsm.edu. URL: http://bgsm.edu/bgsm/biochem/faculty/Poole/poole.html.

${ }^{\otimes}$ Abstract published in Advance ACS Abstracts, October 15, 1997. reagents, we have proposed a catalytic mechanism for the alkyl hydroperoxidase system which includes active-site cysteine residues in both proteins (Scheme 1). In this scheme, AhpF transfers electrons from reduced pyridine nucleotides (especially NADH) to AhpC via the flavin and both redox-active disulfide centers $(6,8)$. Transfer of electrons between $\mathrm{AhpF}$ and $\mathrm{AhpC}$ is envisaged to occur during direct protein-protein interaction that allows for dithiol-disulfide interchange between the two proteins, much like that observed in the thioredoxin reductase-thioredoxin system (9). Reduced AhpC, which contains two thiol groups per subunit, is then proposed to catalyze peroxide reduction through a mechanism closely related to that established for enterococcal NADH peroxidase $(10,11)$; a single cysteine thiolate $\left(\mathrm{Cys}^{-} \mathrm{S}^{-}\right)$attacks the $-\mathrm{O}-\mathrm{O}-$ bond, releasing $\mathrm{ROH}$ and forming a cysteine sulfenic acid (Cys-SOH) within the enzyme. In AhpC, however, this intermediate is expected to be unstable due to the proximity of another cysteine thiol which would react rapidly with the nascent cysteine sulfenic acid to regenerate the disulfide bond and release a molecule of water. In oxidized $\mathrm{AhpC}$, the two half-cystine residues per subunit form intersubunit disulfide bridges as demon-

\footnotetext{
${ }^{1}$ Abbreviations: SDS, sodium dodecyl sulfate; TNB, 2-nitro-5thiobenzoic acid; TSA, thiol-specific antioxidant; DTT, 1,4-dithiothreitol; DTNB, 5,5'-dithiobis(2-nitrobenzoic acid); TPCK, L-1-(tosylamino)2-phenylethyl chloromethyl ketone; HPLC, high-performance liquid chromatography.
} 
Scheme 1

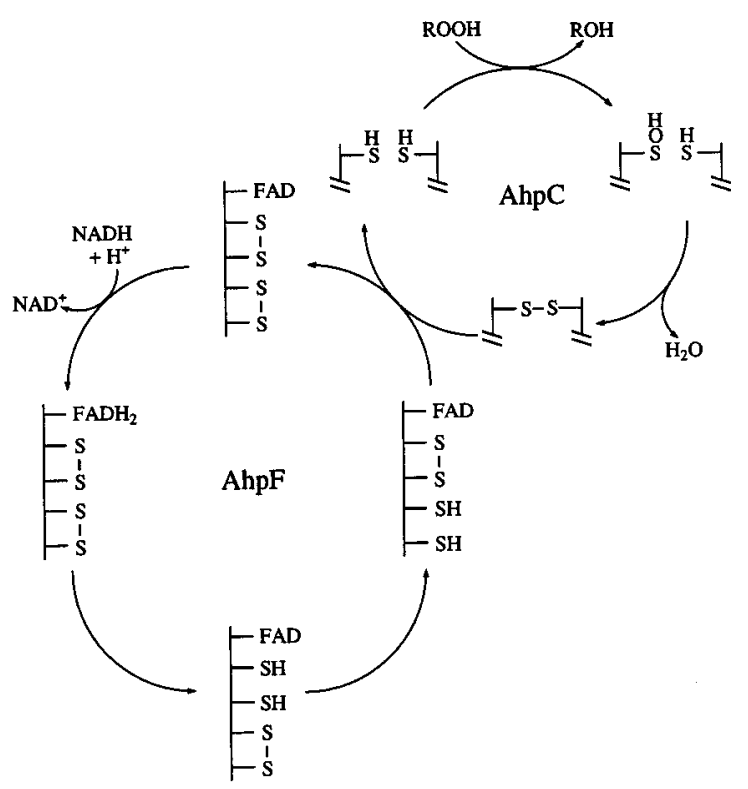

strated by the detection of a single band of dimeric protein on $\mathrm{SDS}^{1}$ - polyacrylamide gels run under nonreducing conditions (5). This intersubunit disulfide bond is highly unusual among proteins where the disulfide acts as a redox center. Such redox centers are usually present as intrasubunit disulfides between half-cystine residues separated by only two or four amino acids (12).

The proposal that a cysteine sulfenic acid is generated, albeit transiently, in AhpC on oxidation of a single cysteine residue by hydroperoxides is well-founded based on the identification of this species within the oxidized form of the flavin-containing NADH peroxidase from Enterococcus faecalis. Studies originally supported the identity of this species in NADH peroxidase through chemical means: the sulfenic acid (1) was reducible by two electrons derived from NADH to generate the cysteine thiol(ate), (2) was susceptible to inactivation by hydrogen peroxide via formation of higher oxidized states of the cysteine, and (3) was reactive with the thiol-containing reagent 2-nitro-5-thiobenzoic acid (TNB) as previously demonstrated for protein-associated sulfenic acids $(10,11)$. Recent studies have directly demonstrated the presence of this species in the oxidized form of NADH peroxidase through cryocrystallography and ${ }^{13} \mathrm{C}-\mathrm{NMR}$ techniques $(13,14)$. A chemically analogous form of selenocysteine, the selenenic acid (Cys-SeOH), has also been identified in the oxidized form of another heme-independent peroxidase protein, glutathione peroxidase (15). This latter protein is similar to AhpC in its broad substrate specificity, reducing hydrogen peroxide as well as bulky hydroperoxides.

A very large family of proteins homologous with AhpC has emerged over the past several years (16) and is now represented by more than 65 different sequences derived from organisms spanning all kingdoms (L. B. Poole, unpublished observations). The majority of these homologues possess cysteine residues corresponding to both Cys46 and Cys165 of S. typhimurium AhpC; Cys46 is conserved among all homologues. Interestingly, it is this cysteine residue that was shown to be essential for antioxidant activity in a yeast homologue, thiol-specific antioxidant (TSA) protein; when the corresponding Cys47 was replaced by Ser, this mutant was unable to protect against the inactivation of glutamine synthetase by a DTT/ $\mathrm{Fe}^{3+} / \mathrm{O}_{2}$ system, while the $\mathrm{C} 170 \mathrm{~S}$ mutant of TSA retained full activity in this protection assay (17).

We describe herein experiments designed to definitively establish the chemical roles of Cys46 and Cys165 in the catalytic mechanism of $S$. typhimurium AhpC using sitedirected mutagenesis, chemical titrations, and activity assays. We also report the identification of the disulfide bond partners in the catalytic redox center of AhpC and provide strong evidence for the participation of a cysteine sulfenic acid in catalytic turnover of this enzyme.

\section{MATERIALS AND METHODS}

Materials. NADH was purchased from BoehringerMannheim. Sigma was the supplier of dimethyl sulfoxide, calcium chloride, streptomycin sulfate, cumene hydroperoxide $(80 \%)$, protocatechuic acid, and protocatechuate 3,4dioxygenase. Hydrogen peroxide (30\%), acetic acid, and Difco bacteriological media were from Fisher. Ultrapure urea, DTNB, SDS, DTT, ammonium bicarbonate, and all buffer components were from Research Organics, Inc. TPCK-treated trypsin was purchased from Worthington Biochemicals. Restriction endonucleases and other DNAmodifying enzymes were purchased from New England Biolabs or Promega and used as recommended by the manufacturer. [ $\left.\alpha-{ }^{35} \mathrm{~S}\right] \mathrm{dATP}$ for DNA sequence analysis was purchased from New England Nuclear. Baker-analyzed HPLC-grade trifluoroacetic acid and acetonitrile were used to prepare solvents for HPLC. The standard buffer used in most experiments was $25 \mathrm{mM}$ potassium phosphate with 1 mM EDTA at $\mathrm{pH} 7$, unless noted otherwise.

Mutagenesis, Cloning, and Purification Procedures. Many of the methods employed in the manipulation of recombinant plasmids and expression and purification of mutant proteins were similar to or identical with those previously described $(5,6)$. To replace Cys46 and Cys165 of AhpC with Ser or Ala in either or both positions, degenerate mutagenic oligonucleotides with the following sequences were designed: 5'-CCTTTGTTT/GCCCCGACTG-3' and 5'-GCGAAGTAT/GCCCCGGCGA-3' (underlined nucleotides are the sites of the new codons, with $\mathrm{T}$ and $\mathrm{G}$ in equal amounts in the first position of these codons; oligonucleotide synthesis was performed in the DNA Synthesis Core Laboratory of the Comprehensive Cancer Center of Wake Forest University supported in part by NIH Grant CA-12197). The $a h p C$ gene for mutagenesis was derived from an EcoRI-EcoRI fragment of pAQ27 cloned into the EcoRI site of M13mp18 $(5,7)$. Single-stranded DNA from this construct was used as template for mutagenesis, and mutant $\mathrm{AhpC}$ constructs were made using the oligonucleotide-directed in vitro mutagenesis kit (version 2.1) from Amersham. The C165S and C46S mutants identified by DNA sequence analysis with the Sequenase 2.0 kit (United States Biochemicals) were subcloned into the Pst $\mathrm{I}-X c m \mathrm{I}$ and $B b s \mathrm{I}-M l u \mathrm{I}$ sites of the pAC1 plasmid, respectively (Figure 1; 5). To create a new expression plasmid, pAC2, these mutant constructs were further subcloned into the PacI-NdeI sites of pACF1, effectively removing the $a h p F$ gene but retaining the OxyRdependent promoter, thus allowing for the expression of the recombinant proteins in a wide variety of host bacteria (Figure 1). The double mutant was constructed by subcloning the 469 bp $M l u \mathrm{I}-N d e \mathrm{I}$ fragment from C165S into the 


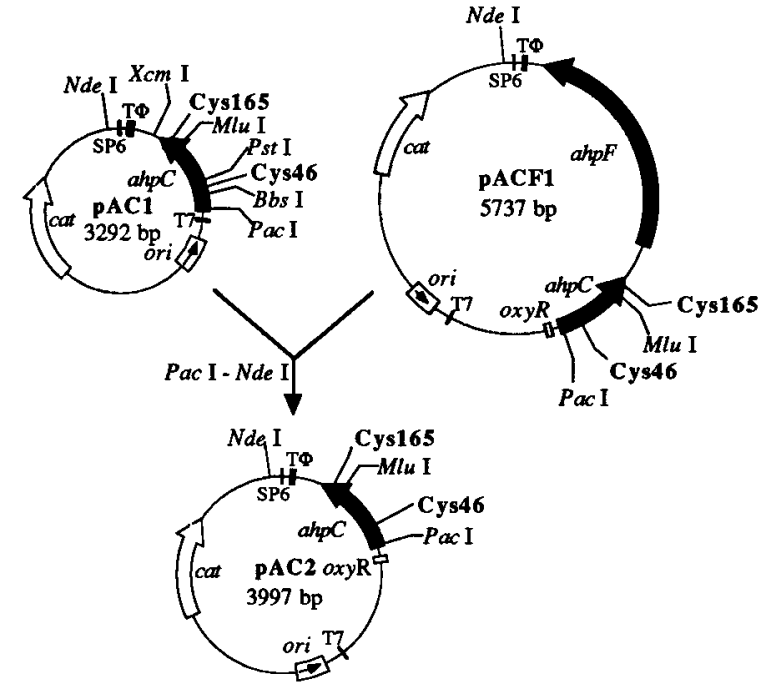

FIGURE 1: Construction of a new expression plasmid for mutant AhpC proteins. The $891 \mathrm{bp} P a c \mathrm{I}-N d e \mathrm{I}$ fragment from $\mathrm{pAC} 1$ was isolated and ligated into the corresponding sites of pACF1 from which the $2.6 \mathrm{~kb} P a c \mathrm{I}-N d e \mathrm{I}$ fragment containing the $a h p F$ gene and most of the $a h p C$ gene had been excised; the latter fragment was dephosphorylated with calf intestinal alkaline phosphatase prior to isolation of both DNA fragments with Gene Clean 2 and overnight ligation with T4 DNA ligase. The desired recombinant plasmids were obtained from competent JM109(DE3) cells transformed with the ligation mixtures and retransformed into the $E$. coli mutant TA4315 for expression as described in the text. T $\Phi$ refers to the $\mathrm{T} 7$ termination sequence from pET-11, cat to the chloramphenicol acetyltransferase gene, and $o x y R$ to the promoter upstream of $a h p C$ recognized by the transcriptional regulator OxyR.

$M l u \mathrm{I}-N d e \mathrm{I}$ sites of the pAC2 plasmid already containing the $\mathrm{C} 46 \mathrm{~S}$ mutation. Following mutagenesis and subcloning, the single $(\mathrm{C} 46 \mathrm{~S}, \mathrm{C} 165 \mathrm{~S})$ and double $(\mathrm{C} 46,165 \mathrm{~S})$ mutant constructs were sequenced in their entirety to verify the mutations and coding sequence, and the recombinant plasmids were transformed into Escherichia coli strain TA4315 which contains a deletion of the $\operatorname{ahpCF}$ operon on the chromosome (3). The proteins were purified as previously described with minor modifications (5). Pelleted bacteria from $24 \mathrm{~L}$ was used for the purification, and in several instances the protein was run a second time on a phenylsepharose 6 Fast Flow column following the DEAE-cellulose column to remove contaminating proteins. In addition, all the buffers used in the purification procedure and the purified mutant proteins contained $5.0 \mathrm{mM}$ DTT to prevent oxidation of the thiols. Proteins were separated from DTT on a Sephadex G-25 column and analyzed for DTNB-reactive thiols prior to further experimentation.

HPLC and Amino Acid Sequence Analyses. Chemical modification and tryptic digestions prior to HPLC analyses were performed as previously described (6). HPLC separations of oxidized and reduced AhpC peptides were carried out on a Rainin HPLC system equipped with a $4.6 \times 100$ mm AquaPore RP-300 C8 column. A gradient of acetonitrile containing $0.1 \%$ trifluoroacetic acid was used for the separation, and the peaks were detected at 215 and $254 \mathrm{~nm}$ on a DynaMax UV-DII dual-wavelength detector. One peptide at $47 \mathrm{~min}$ in the oxidized tryptic map was rechromatographed using a shallower gradient for additional purification. Prior to amino acid sequence analysis, the purified peptide was frozen, lyophilized, and resuspended in $100 \mu \mathrm{L}$ of deionized water. Amino acid sequence analyses were performed in the Protein Analysis Core Laboratory of the Comprehensive Cancer Center of Wake Forest University supported by NIH Grants CA-12197 and PR0048, in addition to a grant from the North Carolina Biotechnology Center.

Mass Spectrometric Analyses. Electrospray ionization mass spectrometric determinations were made on a VG Quattro II triple quadrupole mass spectrometer from Micromass in the GC-Mass Spectrometry Laboratory supported by NSF Grant 9414018 and Comprehensive Cancer Center Grant CA-12197 from NIH. Samples containing $5 \mathrm{nmol}$ of protein were concentrated and rediluted with deionized water 3 times in a Centricon CM-30 at $4000 \mathrm{~g}$ to remove phosphate buffer and EDTA, giving a final volume of approximately $250 \mu \mathrm{L}$. Prior to injection, $250 \mu \mathrm{L}$ of HPLC-grade acetonitrile and $1 \%$ formic acid were added to the sample.

Spectral Experiments. All kinetic assays were performed on an Applied Photophysics DX.17 MV stopped-flow spectrofluorometer thermostatted at $25{ }^{\circ} \mathrm{C}$. The NADH titration and TNB experiments were performed on a thermostatted Milton Roy Spectronic 3000 diode array spectrophotometer with $0.35 \mathrm{~nm}$ resolution. Circular dichroism spectroscopy of the wild-type and mutant $\mathrm{AhpC}$ proteins was performed on a Jasco J-720 spectropolarimeter.

Steady-state aerobic activity assays were carried out on the stopped-flow spectrophotometer by following the oxidation of NADH at $340 \mathrm{~nm}$. Background rates attributed to the relatively low AhpF-dependent oxidase activity were taken into account in calculating specific activities. Assays for the reactivity of free thiols in the reduced wild-type and single AhpC mutants were carried out by reaction with DTNB on the stopped-flow spectrophotometer. The pseudofirst-order rate of reaction was calculated by the increase in $412 \mathrm{~nm}$ corresponding to the formation of TNB.

The anaerobic NADH titrations for single AhpC mutants were performed as previously described (6) with the following exceptions. The proteins were premixed with 1 equiv of hydrogen peroxide following repeated evacuation and nitrogen flushing of the solutions over $20 \mathrm{~min}$. All hydrogen peroxide solutions were standardized by assay with horseradish peroxidase and $o$-dianisidine using a molar extinction coefficient of $11300 \mathrm{M}^{-1} \mathrm{~cm}^{-1}$ at $480 \mathrm{~nm}$ for the oxidized product (18). After mixing with hydrogen peroxide, the solution was flushed for an additional 15 min with nitrogen. $\mathrm{AhpF}$ was added under nitrogen to the bulb of the anaerobic cuvette prior to the addition of the titrating syringe containing an anaerobic solution of NADH.

The TNB reagent was prepared by reacting a slight excess of DTNB with DTT as measured spectrally at $412 \mathrm{~nm}$ (11). Approximately $1 \%$ DTNB was allowed to remain in order to avoid the presence of an excess of DTT. Following the anaerobic reaction of each single mutant of AhpC with 1 equiv of hydrogen peroxide, a 20 -fold excess of TNB was added to the cuvette under nitrogen and then mixed and allowed to react with the protein for $15 \mathrm{~min}$. The reaction with TNB was monitored by the decrease in absorbance at $412 \mathrm{~nm}$. The TNB-modified proteins were then washed four times with deionized water in a Centricon CM-30. Protein recovery after washing, as assessed at $280 \mathrm{~nm}$, was greater than $90 \%$; the conjugate was then reacted with a 10 -fold excess of DTT over protein. The release of TNB was monitored at $412 \mathrm{~nm}$ and the amount of TNB released per subunit was calculated using the extinction coefficient 14150 $\mathrm{M}^{-1} \mathrm{~cm}^{-1}(19)$. 


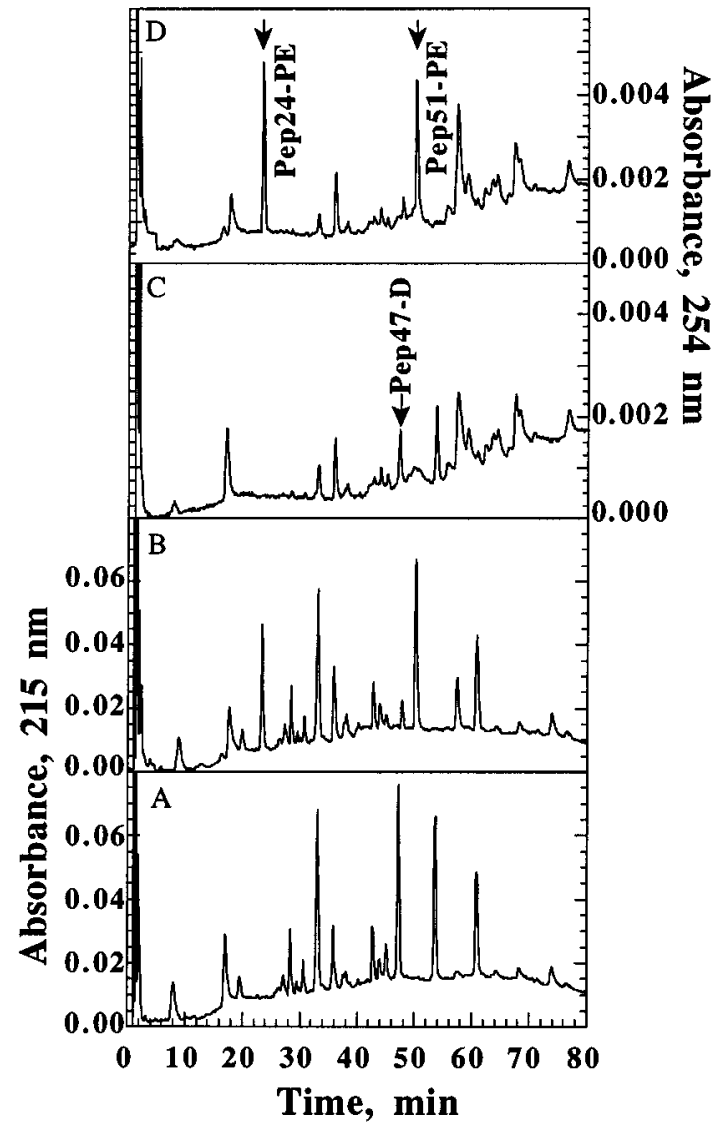

FIGURE 2: HPLC separation of tryptic peptides from oxidized or reduced and pyridylethylated AhpC. Approximately $5 \mathrm{nmol}$ of an exhaustive tryptic digest of oxidized ( $A$ and $C$ ) or reduced, pyridylethylated (B and D) wild-type AhpC was applied to an AquaPore RP-300 C8 column. Solution A contained $0.1 \%$ trifluoroacetic acid, and solution $\mathrm{B}$ contained $70 \%$ acetonitrile and $0.08 \%$ trifluoroacetic acid. After a 5 min wash with $5 \%$ solution B, peptides were eluted with a linear gradient from 5 to $80 \%$ solution B over $80 \mathrm{~min}$ (flow rate was $1 \mathrm{~mL} / \mathrm{min}$ ). Peptide elution was monitored at $215 \mathrm{~nm}$ (panels A and B) and $254 \mathrm{~nm}$ (panels C and D).

\section{RESULTS}

Disulfide Bond Arrangement of Native AhpC. Wild-type AhpC was previously shown to exist as a covalent dimer with two intersubunit disulfide bonds, although the arrangement of these disulfide bonds was not established (5). In order to identify the disulfide linkages present in native AhpC, HPLC-generated tryptic maps of oxidized and reduced, pyridylethylated proteins were compared at 215 and $254 \mathrm{~nm}$. As expected, two peaks exhibiting strong absorbances at $254 \mathrm{~nm}$ were observed in the tryptic map of the reduced, pyridylethylated protein, consistent with those identified and sequenced previously from reduced protein alkylated with iodo $\left[1-{ }^{14} \mathrm{C}\right]$ acetamide (16; Pep24-PE and Pep51-PE in Figure 2B,D). These peaks were absent from the tryptic map of the oxidized AhpC protein, where two other peaks representing potential disulfide-containing peptides were observed at 47 and $54 \mathrm{~min}$ (Figure 2A,C). On isolation of these candidate peptides followed by reduction and pyridylethylation, only the peptide eluting at $47 \mathrm{~min}$ (Pep47-D) was converted to two new peaks corresponding to Pep24-PE and Pep51-PE. Amino acid sequence analysis of Pep47-D confirmed the presence of the disulfide bond between Cys46 and Cys165 in this peptide; two PTH-amino acids were released with each cycle up to the first half-cystine
Table 1: Amino Acid Sequence Analysis of Purified Pep47-D from AhpC

\begin{tabular}{clccc}
\hline cycle & $\begin{array}{c}\text { first } \\
\text { peptide }\end{array}$ & pmol & $\begin{array}{c}\text { second } \\
\text { peptide }\end{array}$ & pmol \\
\hline 1 & Phe & 241 & Val & 300 \\
2 & Phe & 233 & Ala & 381 \\
3 & Tyr & 295 & Ala & 345 \\
4 & Pro & 171 & His & 103 \\
5 & Ala & 338 & Pro & 130 \\
6 & Asp & 311 & Gly & 95 \\
7 & Phe & 163 & Glu & 319 \\
8 & Thr & 194 & Val & 159 \\
9 & Phe & 163 & - & - \\
10 & Val & 174 & Pro & 100 \\
11 & XXX & - & Ala & 168 \\
12 & Pro & 95 & Lys & 58 \\
13 & Thr & 121 & - & - \\
14 & Glu & 47 & - & - \\
\hline
\end{tabular}

${ }^{a}$ Peak eluting at $14.5 \mathrm{~min}$, just after Arg (13.5 min) and Tyr (14.2 $\min$ ); no standard available for identification and quantitation, but this elution time compares favorably with that of the corresponding PTHcystine from AhpF reported previously (6).

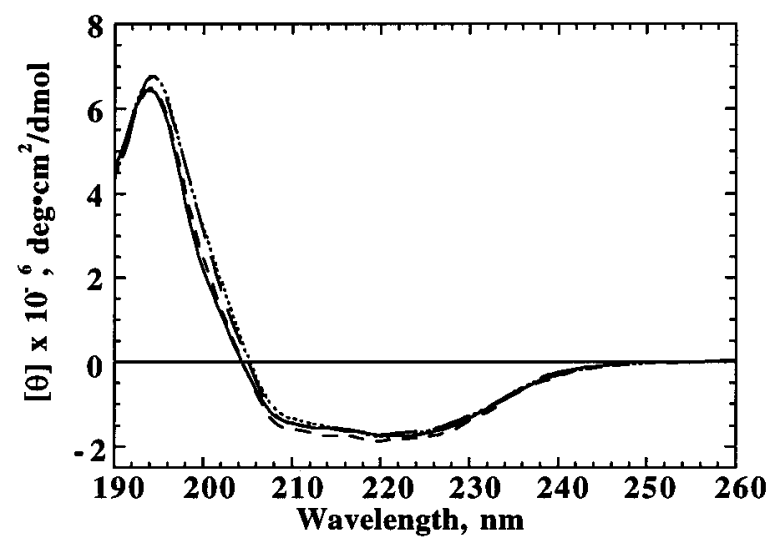

FIGURE 3: Circular dichroism spectroscopy of mutant and wildtype AhpC proteins. Measurements were taken in $0.5 \mathrm{~nm}$ increments from 260 to $190 \mathrm{~nm}$ in a $0.1 \mathrm{~cm}$ path length cuvette with the concentration of protein at $0.2 \mathrm{mg} / \mathrm{mL}$. Each spectrum is the average of four scans; smoothing of the data was performed using the default parameters within the Jasco J-720 software. Spectra shown are for wild-type (solid line), C165S (short dashed line), C46S (long dashed line), and C46,165S (dotted line) AhpC proteins.

encountered (cycle 9), and a peak corresponding to PTHcystine was released from the second peptide in cycle 11 as expected from the sequences (Table 1). These results clearly demonstrate that native AhpC contains two identical intersubunit disulfide bonds per dimer formed between Cys46 and Cys 165 on the other subunit (Cys46-Cys 165'). ${ }^{2}$

Structural Analyses of AhpC Mutants. Substitutions of either or both AhpC cysteine residue(s) by serine residue(s) were made by site-directed mutagenesis in order to further characterize the cysteinyl redox centers of AhpC. Although the replacement of cysteine by serine is generally a conservative substitution, in $\mathrm{AhpC}$ the substitutions should be accompanied by a disruption of the native disulfide bonds present in the oxidized protein. We began our studies by establishing that all cysteine to serine substitutions caused no gross changes in secondary structures within the mutants. As shown in Figure 3, circular dichroism spectra for C46S, C165S, and C46,165S mutants of AhpC are virtually identical

\footnotetext{
${ }^{2}$ Cys $165^{\prime}$ and Cys46' represent cysteine residues located on the opposite subunit when referring to the dimeric arrangement.
} 


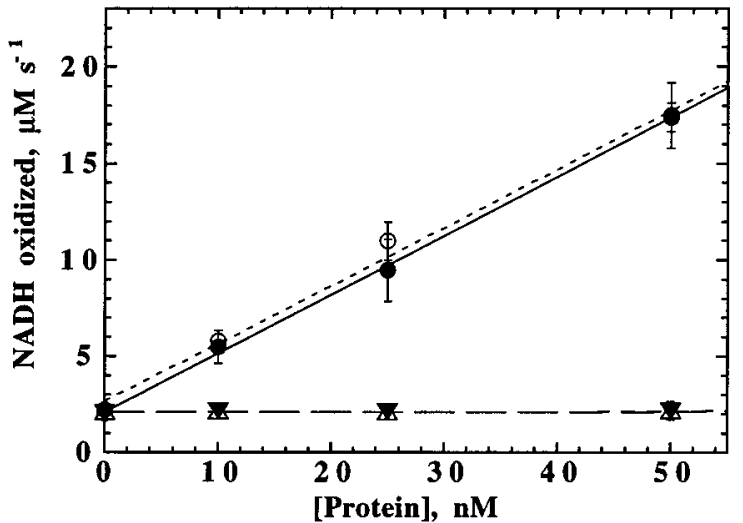

FIGURE 4: Steady-state kinetic analysis of mutant and wild-type AhpC proteins. Stopped-flow spectrophotometric measurements were carried out at $25{ }^{\circ} \mathrm{C}$ with final concentrations of $150 \mathrm{mM}$ ammonium sulfate, $300 \mu \mathrm{M}$ NADH, $1 \mathrm{mM}$ cumene hydroperoxide, $1 \mu \mathrm{M} \mathrm{AhpF}$, and varying concentrations of AhpC (10-100 nM) in $50 \mathrm{mM}$ potassium phosphate buffer with $1 \mathrm{mM}$ EDTA at $\mathrm{pH}$ 7.0. Reactions were initiated on mixing the proteins in one syringe with the substrates in the other syringe. Data shown are from three separate experiments with wild-type (closed circles), C165S (open circles), C46S (inverted closed triangles), and C46,165S (open triangles) AhpC proteins. Rates were determined by linear regression analysis of the change in absorbance at $340 \mathrm{~nm}$ from the first $3 \mathrm{~s}$ after mixing.

in the far-UV region with that of the reduced wild-type AhpC protein. Although not shown, there is also no significant difference between circular dichroism spectra of oxidized and reduced forms of wild-type AhpC.

As expected from the results described in the previous section, each mutation of a cysteine residue removed the intersubunit disulfide bridges observed in the wild-type protein. Electrospray ionization mass spectrometry indicated a molecular mass value of $41229.34 \pm 1.91$ amu for wildtype AhpC (compared with an expected value of $41228 \mathrm{amu}$ for the dimeric protein, calculated as twice the monomeric value minus 4 hydrogens per dimer accounting for disulfide formation; 5). Single mutants (C165S and C46S) gave values of $20599.88 \pm 0.57$ and $20596.42 \pm 0.66 \mathrm{amu}$, respectively, compared to expected values of $20600 \mathrm{amu}$ for each mutant in its monomeric form. The molecular mass of the double mutant $(\mathrm{C} 46,165 \mathrm{~S})$ was $20582.31 \pm 0.76 \mathrm{amu}$, which is in agreement with the expected value of 20584 amu for the monomeric form of this mutant.

Steady-State Kinetic Analyses of AhpC Mutants. In order to address the requirement for one or both cysteine residues in the catalysis of peroxide reduction, single (C46S and C165S) and double (C46,165S) mutants of AhpC were tested for activity in the presence of excess AhpF using a stoppedflow spectrophotometer. We have found that initial rates obtained in this manner are far greater (by 30-fold or more) than those previously reported which employed anaerobic cuvettes and manual mixing times of $>30 \mathrm{~s}$ (5). Further analyses comparing the two methods have indicated that wild-type AhpC is sensitive to inactivation by peroxide substrates during turnover, and that this inactivation rate is highly sensitive to the reaction conditions used. In the present work, we have utilized the stopped-flow spectrophotometer for all steady-state assays and used data obtained during the first $3 \mathrm{~s}$ after mixing, a method which gives far more reproducible results than those previously reported (5).

As shown in Figure 4, NADH oxidation rates were nearly identical for wild-type and C165S AhpC proteins, at $318 \pm$
21 and $348 \pm 30 \mathrm{nM}$ NADH oxidized $\mathrm{s}^{-1} \mathrm{nM}^{-1}$ protein, respectively, and were directly proportional to the amount of AhpC added. In contrast, neither the C46S nor the C46,165S mutant exhibited activity under these conditions (Figure 4). The non-zero intercept represents the relatively low NADH oxidase activity catalyzed by AhpF alone. These results clearly implicate the thiol of Cys 46 as the peroxidatic center in the active site of AhpC.

As demonstrated in Figure 4, the C165S mutant of AhpC exhibits full peroxidatic activity in the presence of $1 \mu \mathrm{M}$ $\mathrm{AhpF}$; in contrast, little or no peroxidatic activity of $\mathrm{C} 165 \mathrm{~S}$ is observed if $\mathrm{AhpF}$ is present at concentrations below 200 $\mathrm{nM}$ (data not shown). This inactivating effect of lowering $\mathrm{AhpF}$ concentrations is not due to the presence of oxygen; results are identical when the same experiments are carried out under anaerobic conditions. These results are readily explained if cysteine sulfenic acid is formed on oxidation of Cys46 by the hydroperoxide substrate. Whether this reactive intermediate is further oxidized to sulfinic or sulfonic acid forms, and thus inactivated by the excess cumene hydroperoxide present, or is re-reduced by $\mathrm{AhpF}$ to continue the catalytic cycle appears to be a function of the amount of $\mathrm{AhpF}$ available in the reaction mixture. Wild-type AhpC is not similarly sensitive to lowered $\mathrm{AhpF}$ concentrations, presumably due to the presence of the proximal Cys $165^{\prime}$ thiol within the active site which rapidly re-forms the highly stable disulfide bond with Cys46.

$\mathrm{NADH}$ Titrations of AhpC Mutants Pretreated with $\mathrm{H}_{2} \mathrm{O}_{2}$. We set out to examine the species generated on oxidation of each single mutant of AhpC by 1 equiv of $\mathrm{H}_{2} \mathrm{O}_{2}$ using reductive titrations with $\mathrm{NADH}$. These titrations were performed in the presence of catalytic amounts of AhpF, monitoring the absorbance at $340 \mathrm{~nm}$ as NADH was added anaerobically from a titrating syringe. Previous experiments demonstrated the consumption of 1 equiv of $\mathrm{NADH}$ and the generation of two new thiol groups per subunit of AhpC under similar conditions (6). In order to generate the putative oxidized forms of the single cysteine-containing AhpC mutants, preparations of $\mathrm{C} 46 \mathrm{~S}$ or $\mathrm{C} 165 \mathrm{~S}$ from which DTT had been removed were preincubated with 1 equiv of $\mathrm{H}_{2} \mathrm{O}_{2}$ under anaerobic conditions for 15 min prior to the addition of $\mathrm{AhpF}$ and anaerobic titration with NADH. As shown in Figure 5, 1 equiv of NADH was consumed per subunit during titrations of the preincubated C165S AhpC mutant, similar to the result observed with wild-type AhpC, while titrations of the similarly-treated C46S mutant resulted in no consumption of NADH, giving only a steady increase in the $340 \mathrm{~nm}$ absorbance throughout the experiment. DTNB assays of the two single mutants confirmed the presence of a single thiol group per subunit following titration of either mutant by $\mathrm{NADH}$, while only the $\mathrm{H}_{2} \mathrm{O}_{2}$-treated $\mathrm{C} 46 \mathrm{~S}$ mutant reacted with DTNB prior to the titration with NADH. Additional experiments with the $\mathrm{C} 46 \mathrm{~S}$ mutant indicated that neither lengthening the time of preincubation with $\mathrm{H}_{2} \mathrm{O}_{2}$ (up to 120 min) nor increasing the $\mathrm{H}_{2} \mathrm{O}_{2}$ concentration (up to a 100fold excess) brought about any significant change in the titration results with this mutant. Clearly only the $\mathrm{C} 165 \mathrm{~S}$ mutant is stoichiometrically oxidized by $\mathrm{H}_{2} \mathrm{O}_{2}$ to a form which can be efficiently reduced by AhpF with two electrons derived from NADH to regenerate the Cys46 thiol of the reduced, active protein.

TNB Reactivity of Oxidized C165S AhpC. As the NADH titration described above provided evidence of an oxidized 


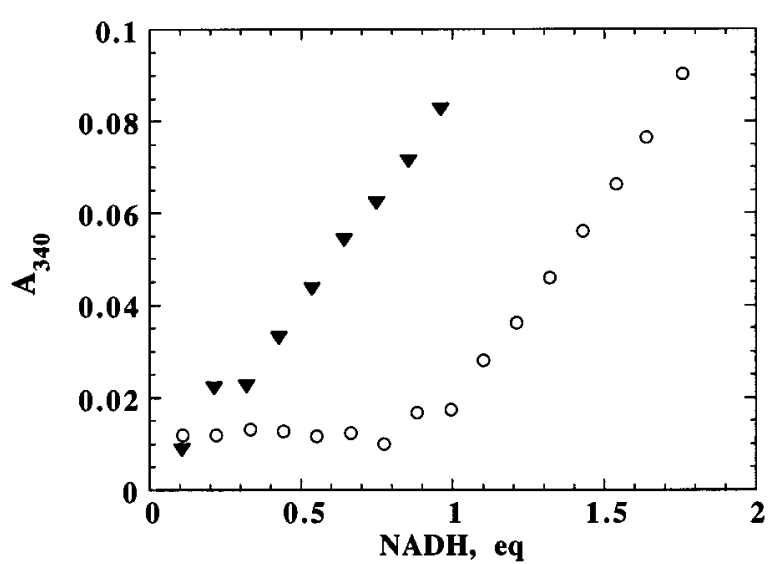

FIGURE 5: Anaerobic NADH titrations of $\mathrm{H}_{2} \mathrm{O}_{2}$-treated C46S and C165S mutants of AhpC in the presence of AhpF. AhpC mutants (20 nmol) were reacted for 15 min with 1 equiv of hydrogen peroxide under anaerobic conditions in $0.6 \mathrm{~mL}$ of a solution containing $50 \mathrm{mM}$ potassium phosphate and $1 \mathrm{mM}$ EDTA at $\mathrm{pH}$ 7.0 in the presence of protocatechuate dioxygenase ( 0.02 unit) and protocatechuic acid $(20 \mathrm{nmol})$ to eliminate oxygen. Spectra were collected after all changes were complete following each addition of the anaerobic NADH solution from a titrating syringe. Shown is the absorbance monitored at $340 \mathrm{~nm}$ versus equivalents of NADH added relative to the $\mathrm{AhpC}$ monomer for $\mathrm{H}_{2} \mathrm{O}_{2}$-treated $\mathrm{C} 46 \mathrm{~S}$ (inverted closed triangles) and $\mathrm{C} 165 \mathrm{~S}$ (open circles) proteins. As shown, the oxidized C165S mutant required 1.01 NADH/AhpC monomer for complete reduction; results from three experiments gave an average of $1.09 \pm 0.07 \mathrm{NADH} / \mathrm{AhpC}$ monomer.

species of Cys 46 consistent with the redox behavior of a cysteine sulfenic acid, we sought independent evidence for this species through measurement of its reactivity with a chromophoric thiol-containing reagent, TNB. The C165S mutant of AhpC was preincubated with 1 equiv of $\mathrm{H}_{2} \mathrm{O}_{2}$ under anaerobic conditions as described above, and then reacted with TNB while monitoring the absorbance at 412 $\mathrm{nm}$. The decrease in $A_{412}$ accounted for $0.86 \pm 0.08 \mathrm{TNB}$ consumed per subunit of C165S. As expected from the NADH titration experiments described above, no decrease in $A_{412}$ was observed on addition of TNB to the C46S mutant pretreated with $\mathrm{H}_{2} \mathrm{O}_{2}$. An additional measure of the TNB reaction stoichiometry was obtained on reaction of the isolated TNB conjugate of C165S with DTT. Figure 6 demonstrates that the reduction of the conjugate proceeded with a $t_{1 / 2}$ of about $2.1 \mathrm{~min}$ and gave a total of $0.81 \pm 0.06$ equiv of TNB released per mole of protein. Again, no TNB was released from the C46S mutant treated in a similar manner. Thus, the reactivity of the oxidized form of C165S is consistent with that previously established for cysteine sulfenic acids (11).

In order to address the possibility that the $\mathrm{p} K_{\mathrm{a}}$ of the thiol of Cys 165 was substantially higher than that of Cys46 and responsible for the lack of reactivity of this residue toward $\mathrm{H}_{2} \mathrm{O}_{2}$, the TNB experiments with $\mathrm{H}_{2} \mathrm{O}_{2}$-treated mutants described above were repeated at $\mathrm{pH}$ 9.0. Again, TNB reacted stoichiometrically with $\mathrm{C} 165 \mathrm{~S}$ but did not give detectable reaction with $\mathrm{C} 46 \mathrm{~S}$. Denaturation of each of the mutants with guanidine hydrochloride prior to $\mathrm{H}_{2} \mathrm{O}_{2}$ treatment and TNB modification indicated that both mutants were unreactive toward $\mathrm{H}_{2} \mathrm{O}_{2}$ at $\mathrm{pH}$ 7.0, and partially reactive at $\mathrm{pH}$ 9.0. These results clearly implicate the microenvironment of each of the cysteine residues in modulating their reactivity toward $\mathrm{H}_{2} \mathrm{O}_{2}$, as discussed below.

DTNB Reactivity of AhpC Mutants. One explanation for the differential reactivity of the AhpC C46S and C165S

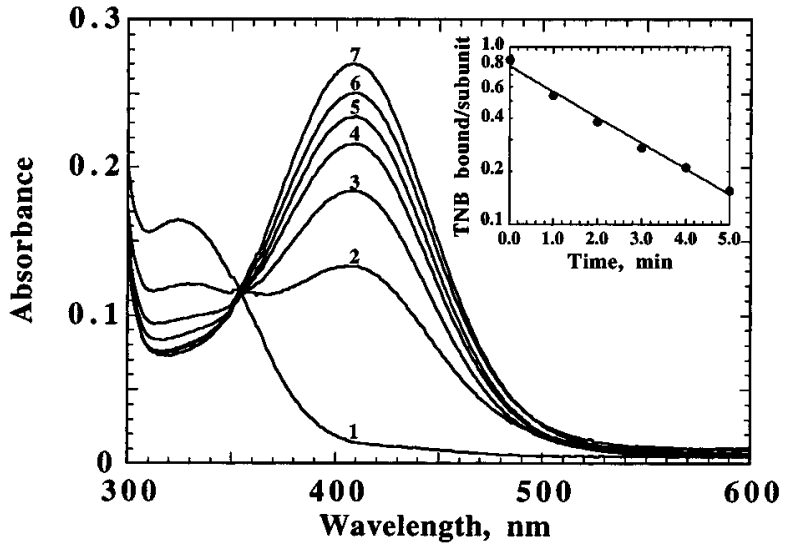

FIGURE 6: Reduction of the AhpC/C165S-TNB conjugate with DTT. The C165S-TNB conjugate was generated by mixing 20 nmol of C165S pretreated anaerobically with 1 equiv of hydrogen peroxide in a $0.6 \mathrm{~mL}$ solution of the standard buffer with $400 \mathrm{nmol}$ of TNB. Following removal of free TNB from the solution in a Centricon CM-30, a 10-fold excess of DTT was added to the modified protein and the spectral changes were recorded as follows: 1, spectrum of the $\mathrm{C} 165 \mathrm{~S}-\mathrm{TNB}$ conjugate prior to the addition of DTT; 2-7, spectra at 1, 2, 3, 4, 5, and 30 min after the addition of DTT, respectively. The inset is a semilogarithmic plot of the change in absorbance, converted into units of TNB/subunit, versus time.

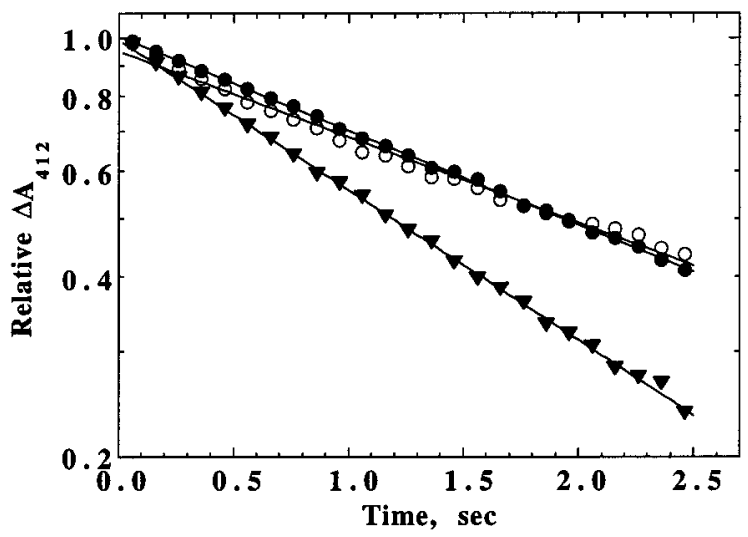

FIGURE 7: DTNB reactivity of reduced wild-type, C46S, and C165S AhpC proteins. Stopped-flow measurements were made at $25{ }^{\circ} \mathrm{C}$ in the standard phosphate buffer at $\mathrm{pH} 7.0$ upon mixing $\mathrm{AhpC}$ protein and DTNB at final concentrations of $20 \mu \mathrm{M}$ and $400 \mu \mathrm{M}$, respectively. The rate of TNB production was measured at $412 \mathrm{~nm}$ for a total of $20 \mathrm{~s}$. The $y$-axis represents the total absorbance change normalized to 1.0 for all proteins. The rates were determined from two separate experiments and are depicted for the single C46S (inverted closed triangles) and $\mathrm{C} 165 \mathrm{~S}$ (open circles) mutants and wild-type (closed circles) AhpC.

cysteine thiol groups toward $\mathrm{H}_{2} \mathrm{O}_{2}$ might be a difference in the accessibility of these groups toward modifying agents. We sought to test this hypothesis through analyses of reaction rates of the thiol group from each mutant with the chromophoric thiol reagent DTNB. Shown in Figure 7 are the absorbance changes observed at $412 \mathrm{~nm}$ on addition of this reagent to $\mathrm{C} 46 \mathrm{~S}, \mathrm{C} 165 \mathrm{~S}$, and reduced wild-type $\mathrm{AhpC}$; pseudo-first-order rate constants for these spectral changes were $0.60,0.45$, and $0.47 \mathrm{~s}^{-1}$, respectively. As expected, the extent of each reaction accounted for the modification of $0.92 \pm 0.03,1.0 \pm 0.04$, and $1.89 \pm 0.06$ thiols/subunit, respectively. Thus, the active single mutant of $\mathrm{AhpC}$, C165S, reacts with DTNB at a rate nearly identical with that of the thiol groups of the wild-type protein (previous results have demonstrated that only one thiol of reduced, native AhpC reacts with DTNB; the second thiol attacks the mixed 
disulfide bond to release a second TNB anion and re-form the native intersubunit disulfide bond; 6 ). The AhpC mutant which appears to be unreactive with $\mathrm{H}_{2} \mathrm{O}_{2}$ or cumene hydroperoxide, $\mathrm{C} 46 \mathrm{~S}$, is actually slightly more reactive toward DTNB, eliminating inaccessibility as an argument for the lack of reactivity of the Cys165 thiol toward $\mathrm{H}_{2} \mathrm{O}_{2}$.

\section{DISCUSSION}

The results described herein have established the arrangement of the unusual intersubunit disulfide bonds which act as catalytic centers in AhpC, and have clarified the chemical roles for each cysteine residue within the active site. Through amino acid sequence analyses of the disulfidecontaining tryptic peptide, the AhpC subunits were shown to exist in an antiparallel arrangement, with Cys46 forming a disulfide bond with Cys165 on the other subunit. The antiparallel arrangement was further supported by the mass spectrometric data; the molecular mass obtained for each of the single mutants is for the monomeric form while the mass obtained for wild-type $\mathrm{AhpC}$ is for the dimeric form of the protein. If the subunit arrangement were parallel (Cys46Cys46' and Cys165-Cys165'), each single mutant should still be able to form one disulfide bond per dimer. Our results with AhpC are in agreement with those for a related protein, TSA from yeast, which was also shown to possess intersubunit disulfide bonds oriented primarily in this antiparallel fashion (17). These results strongly suggest that there are two identical active sites per dimer in the AhpC protein.

In order to better understand the function of each cysteine residue in catalysis, single $(\mathrm{C} 46 \mathrm{~S}$ and $\mathrm{C} 165 \mathrm{~S})$ and double (C46,165S) cysteine to serine mutants were constructed. These proteins were expressed in E. coli strain TA4315 which contains a deletion of the $\operatorname{ahpCF}$ operon and thus allows for the expression of these proteins without any wildtype background. Circular dichroism spectra of the mutants were compared with that of wild-type to verify that no gross secondary structural changes had occurred. Mass spectrometry of the single and double mutant proteins, in addition to DNA sequencing of the full coding sequences of the expression vectors and quantitation of the thiol contents of each protein, confirmed that the desired mutations were present and unaccompanied by any other inadvertent mutations.

Catalytic functions of AhpC mutants possessing either one or no cysteine residues per subunit were assessed by activity assays carried out in the presence of excess $\mathrm{AhpF}$ using a stopped-flow spectrophotometer. Our previous demonstration that reduced AhpC was very sensitive to inactivation by thiol reagents led to the expectation that $\mathrm{C} 46,165 \mathrm{~S}$, which contains no cysteine residues, would be totally inactive. We did recognize, however, that the presence of only one cysteine per subunit in AhpC could still be sufficient to support peroxide reduction during turnover, as is the case in the active site of the single cysteine-containing NADH peroxidase from $E$. faecalis $(10,11)$. The difference for the active single mutant versus wild-type AhpC would be that $\mathrm{AhpF}$ would have to reduce the putative cysteine sulfenic acid generated on oxidation of the catalytic cysteine rather than the disulfide bond of wild-type AhpC (Scheme 2). Evaluation of the catalytic activities of wild-type and mutant proteins indicated that one $(\mathrm{C} 165 \mathrm{~S})$, but not the other $(\mathrm{C} 46 \mathrm{~S})$,
Scheme 2

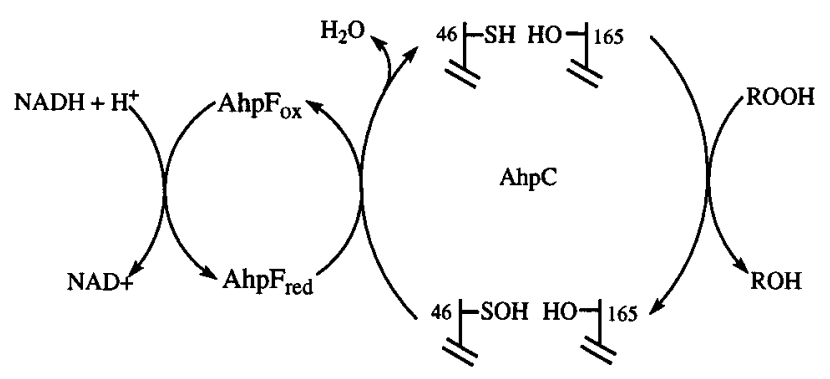

single mutant of AhpC exhibited full peroxidatic activity, while the double mutant was completely inactive. This result demonstrates that Cys46 is absolutely necessary for catalysis of peroxide reduction by AhpC.

Further experimentation with the C165S mutant of AhpC indicated that the activity of this mutant is highly dependent on the reaction conditions. When $\mathrm{AhpF}$ concentrations lower than $200 \mathrm{nM}$ were used, C165S exhibited little or no activity toward hydroperoxide substrates, apparently due to inactivation of the protein by excess peroxide. The oxidized form of $\mathrm{C} 165 \mathrm{~S}$ is also susceptible to inactivation by oxygen if exposed to air over several minutes (data not shown), but this autoxidation appears not to be of consequence when the C165S mutant is actively turning over.

Our interpretation of the above results is that the ability of $\mathrm{C} 165 \mathrm{~S}$ to catalyze continuous peroxide reduction is dependent on having the levels of reductants (AhpF and $\mathrm{NADH}$ ) sufficiently high to promote reduction of the cysteine sulfenic acid (Cys46-SOH) back to the thiol (Cys46-SH) rather than oxidation of this center to sulfinic $\left(\mathrm{Cys} 46-\mathrm{SO}_{2} \mathrm{H}\right)$ or sulfonic $\left(\mathrm{Cys} 46-\mathrm{SO}_{3} \mathrm{H}\right)$ acid forms by excess peroxide. Peroxide-mediated inactivation of the enterococcal NADH peroxidase was also attributable to the generation of these higher oxidation states of the catalytic cysteine residue (11). These findings also support a similar interpretation of the contrasting results obtained from activity measurements of the comparable C170S mutant of yeast TSA. In those experiments, the mutant exhibited full "protector" activity in the presence of DTT, preventing inactivation of glutamine synthetase activity by a metal-catalyzed oxidation system (DTT/ $/ \mathrm{Fe}^{3+} / \mathrm{O}_{2}$ ), but no peroxidase activity in the presence of NADPH, thioredoxin, and thioredoxin reductase $(17,20$, 21). The efficiency of reduction of the C170S mutant of TSA by the thioredoxin reductase system may not be sufficient to prevent peroxide-mediated inactivation of this mutant.

These findings also explain our previous in vivo results which indicated that none of these AhpC mutants, C46S, $\mathrm{C} 165 \mathrm{~S}$, or $\mathrm{C} 46,165 \mathrm{~S}$, provided detectable protection of host bacteria against the cytotoxic effects of cumene hydroperoxide (22). In similar survival assays showing complementation of an $a h p C$-deficient $E$. coli strain by Mer5, a mouse homologue of AhpC, a mutation of the second cysteine corresponding to Cys165 in S. typhimurium did still result in a moderate amount of protection against peroxide treatment relative to wild-type sense and antisense strains (23). Thus, these studies did indicate a detectable amount of activity in a "conditionally active mutant" tested in vivo; it is notable that this partially active Mer5 mutant corresponds to our C165S AhpC mutant which also exhibits peroxidase activity. Taken together, our results indicate that although 
Cys165 is not directly required for the reduction of hydroperoxides, it does play an important role in preventing the inactivation of $\mathrm{AhpC}$ through reversible formation of a stable cystine disulfide bond with the nascent $\mathrm{Cys} 46-\mathrm{SOH}$ at the active site.

An important result of these studies is that the inactive single mutant of AhpC, C46S, does not react with hydrogen peroxide even when this oxidant is added in excess. Reactivity of Cys 165 toward DTNB is at least as great as that of Cys46, eliminating inaccessibility as a factor in the inability of Cys 165 to react with $\mathrm{H}_{2} \mathrm{O}_{2}$. One alternative explanation for this lack of reactivity would be that the $\mathrm{p} K_{\mathrm{a}}$ of the Cys165 thiol group is substantially higher than that of Cys46 due to differences in their microenvironments. This explanation is not, however, supported by the similar reaction rates for the cysteine thiol(ate)s of the single mutants with DTNB, a result which suggests a similar degree of ionization, and therefore nucleophilicity, for these cysteinyl residues (24). The best explanation for the differential reactivities of Cys46 and Cys165 toward peroxides may be their respective orientations relative to an active-site base which could assist in proton donation to the poor $\mathrm{RO}^{-}$leaving group of the peroxide substrate, a feature that would be unnecessary for TNB formation due to the high stability of this anion at neutral $\mathrm{pH}$. Further investigations to determine the threedimensional structure of AhpC and to identify such a putative active-site residue will be required to test this hypothesis.

Finally, we have presented strong evidence that cysteine sulfenic acid is the intermediate formed on reaction of the catalytic cysteine residue, Cys46, with $\mathrm{H}_{2} \mathrm{O}_{2}$. Although this species cannot be detected in the wild-type protein due to its probable rapid condensation with the proximal thiol(ate) of Cys165 in the active site, it is sufficiently stable under anaerobic conditions in the $\mathrm{C} 165 \mathrm{~S}$ mutant to be titrated back to the thiol form by NADH (in the presence of AhpF) and to be quantitated through reaction with TNB. This species is also susceptible to further peroxide- and oxygen-mediated oxidation which leads to inactivation of the C165S mutant. These chemical characteristics match those of the known cysteine sulfenic acid which comprises the active-site redox center of enterococcal NADH peroxidase $(10,11)$. The catalytic mechanism for peroxide reduction by $\mathrm{AhpC}$ is thus fully analogous to that of NADH peroxidase and the selenocysteine-containing glutathione peroxidase, with an additional step of condensation of the nascent oxidized cysteine, Cys46-SOH, with Cys165'-SH to regenerate the active-site disulfide bond and thus stabilize the oxidized protein.

\section{ACKNOWLEDGMENT}

We thank Lois LaPrade for technical assistance, Conn Mallett for generating C46S and C165S mutants and sub- cloning them into pAC1, Al Claiborne and Jeff Schmitt for valuable discussions, and the reviewers of the manuscript for helpful suggestions regarding the interpretation of our data.

\section{REFERENCES}

1. Christman, M. F., Morgan, R. W., Jacobson, F. S., and Ames, B. N. (1985) Cell 41, 753-762.

2. Jacobson, F. S., Morgan, R. W., Christman, M. F., and Ames, B. N. (1989) J. Biol. Chem. 264, 1488-1496.

3. Storz, G., Jacobson, F. S., Tartaglia, L. A., Morgan, R. W., Silveira, L. A., and Ames, B. N. (1989) J. Bacteriol. 171, 2049-2055.

4. Poole, L. B. (1993) in Flavins and Flavoporoteins (Yagi, K., Ed.) pp 583-586, Walter de Gruyter, New York.

5. Poole, L. B., and Ellis, H. R. (1996) Biochemistry 35, 5664.

6. Poole, L. B. (1996) Biochemistry 35, 65-75.

7. Tartaglia, L. A., Storz, G., Brodsky, M. H., Lai, A., and Ames, B. N. (1990) J. Biol. Chem. 265, 10535-10540.

8. Li Calzi, M., and Poole, L. B. (1997) Biochemistry 36, 1335713364.

9. Williams, C. H., Jr. (1992) in Chemistry and Biochemistry of Flavoenzymes (Müller, F., Ed.) pp 121-211, CRC Press, Inc., Boca Raton, FL.

10. Poole, L. B., and Claiborne, A. (1989) J. Biol. Chem. 264, $12322-12329$.

11. Poole, L. B., and Claiborne, A. (1989) J. Biol. Chem. 264, 12330-12338.

12. Williams, C. H., Jr. (1976) in The Enzymes (Boyer, P. D., Ed.) pp 89-173, Academic Press, New York.

13. Yeh, J. I., Claiborne, A., and Hol, W. G. J. (1996) Biochemistry 35, 9951-9957.

14. Crane, E. J., III, Vervoort, J., and Claiborne, A. (1997) Biochemistry 36, 8611-8618.

15. Epp, O., Ladenstein, R., and Wendel, A. (1983) Eur. J. Biochem. 133, 51-69.

16. Chae, H. Z., Robison, K., Poole, L. B., Church, G., Storz, G., and Rhee, S. G. (1994) Proc. Natl. Acad. Sci. U.S.A. 91, 70177021.

17. Chae, H. Z., Uhm, T. B., and Rhee, S. G. (1994) Proc. Natl. Acad. Sci. U.S.A. 91, 7022-7026.

18. Worthington Enzyme Manual (1972) pp 41-45, Worthington Biochemical Corporation, Freehold, NJ.

19. Riddles, P. W., Blakeley, R. L., and Zerner, B. (1979) Anal. Biochem. 94, 74-81.

20. Chae, H. Z., Chung, S. J., and Rhee, S. G. (1994) J. Biol. Chem. 269, 27670-27678.

21. Netto, L. E. S., Chae, H. Z., Kang, S.-W., Rhee, S. G., and Stadtman, E. R. (1996) J. Biol. Chem. 271, 15315-15321.

22. Ellis, H. R., and Poole, L. B. (1995) FASEB J. 9, A592.

23. Tsuji, K., Copeland, N. G., Jenkins, N. A., and Obinata, M. (1995) Biochem. J. 307, 377-381.

24. Dahl, K. H., and McKinley-McKee, J. S. (1981) Bioorg. Chem. 10, 329-341.

BI9713658 